\title{
An unusual cause of back pain in osteoporosis: lessons from a spinal lesion
}

\author{
S Venkatachalam, Elaine Dennison, Madeleine Sampson, Peter Hockey, M I D Cawley, \\ Cyrus Cooper
}

\section{Case report}

A 77 year old woman was admitted with a three month history of worsening back pain, malaise, and anorexia. On direct questioning, she reported that she had suffered from back pain for four years. The thoracolumbar radiograph four years earlier showed T6/7 vertebral collapse, mild scoliosis, and degenerative change of the lumbar spine (fig 1); but other investigations at that time including the erythrocyte sedimentation rate (ESR) and protein electophoresis were normal. Bone mineral density then was $0.914 \mathrm{~g} / \mathrm{cm}^{2}($ T score $=-2.4)$ at the lumbar spine, $0.776 \mathrm{~g} / \mathrm{cm}^{2}(\mathrm{~T}$ score $=$ $-1.8)$ at the right femoral neck and $0.738 \mathrm{~g} / \mathrm{cm}^{2}$ ( $\mathrm{T}$ score $=-1.7$ ) at the left femoral neck. She was given cyclical etidronate after this vertebral collapse as she had suffered a previous fragility fracture of the left wrist.

On admission, she was afebrile, but general examination was remarkable for pallor, dental caries, and cellulitis of the left leg. A pansystolic murmur was heard at the cardiac apex on auscultation; there were no other signs of bacterial endocarditis. She had kyphoscoliosis and there was diffuse tenderness of the thoracolumbar spine. Her neurological examination was unremarkable.

Laboratory investigations showed a marked normocytic anaemia $(\mathrm{Hb}, 6.6 \mathrm{~g} / \mathrm{dl}, \mathrm{MCV}, 80$ fl), leucocytosis (TLC, $19.9 \times 10^{9} / 1$, neutrophils, 12.1), ESR of $45 \mathrm{~mm} \mathrm{1st} \mathrm{hour,} \mathrm{and} \mathrm{C}$ reactive protein (CRP) of $89.9 \mathrm{mg} / 1$ (normal<6 $\mathrm{mg} / \mathrm{l}$ ). There was polyclonal increase of immunoglobulin; biochemical profile and urine analysis were normal. Blood cultures grew Streptococcus mitis (alpha-haemolytic) and an echocardiogram showed a large vegetation on the posterior mitral leaflet, confirming the clinical suspicion of bacterial endocarditis. After microbiological review, she was given intravenous teicoplanin (because of penicillin allergy) and netilmycin. A blood transfusion was also arranged.

The back pain gave cause for concern as she found it difficult to turn in bed. New radiographs showed an increase in the scoliosis, besides the previously recorded vertebral deformities (T6/7) and degenerative change. Technetium $\left({ }^{99 \mathrm{~m}} \mathrm{Tc}\right)$ scintigraphy showed increased uptake in the thoracolumbar spine, consistent with osteoporosis and osteoarthritis.

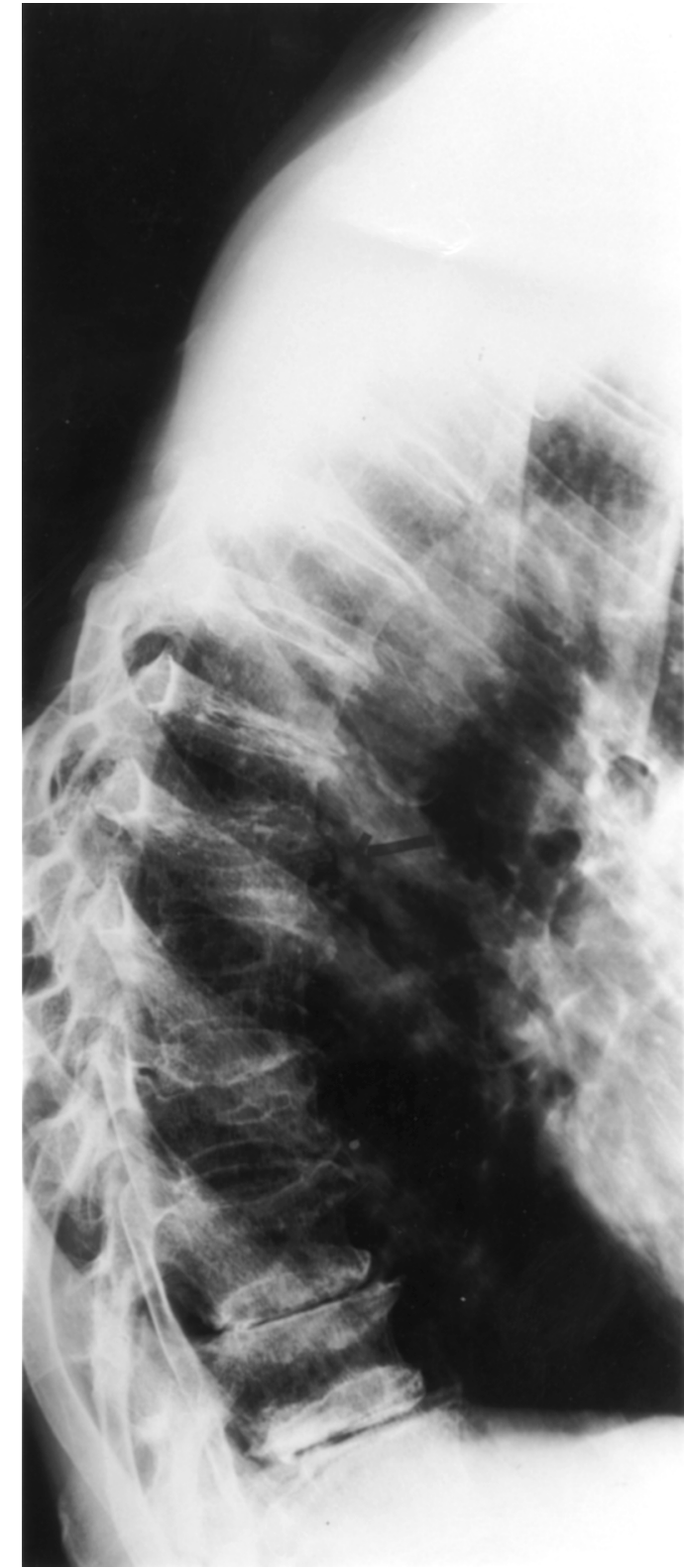

Figure 1 Lateral radiograph of the thoracic spine showing T6/7 vertebral collapse.

She was treated with opioid analgesics and subcutaneous calcitonin injections and supported with a corset. In the fourth week of her inpatient stay, she suddenly developed weakness of the legs, loss of sensation up to T8, and incontinence. Magnetic resonance imaging 


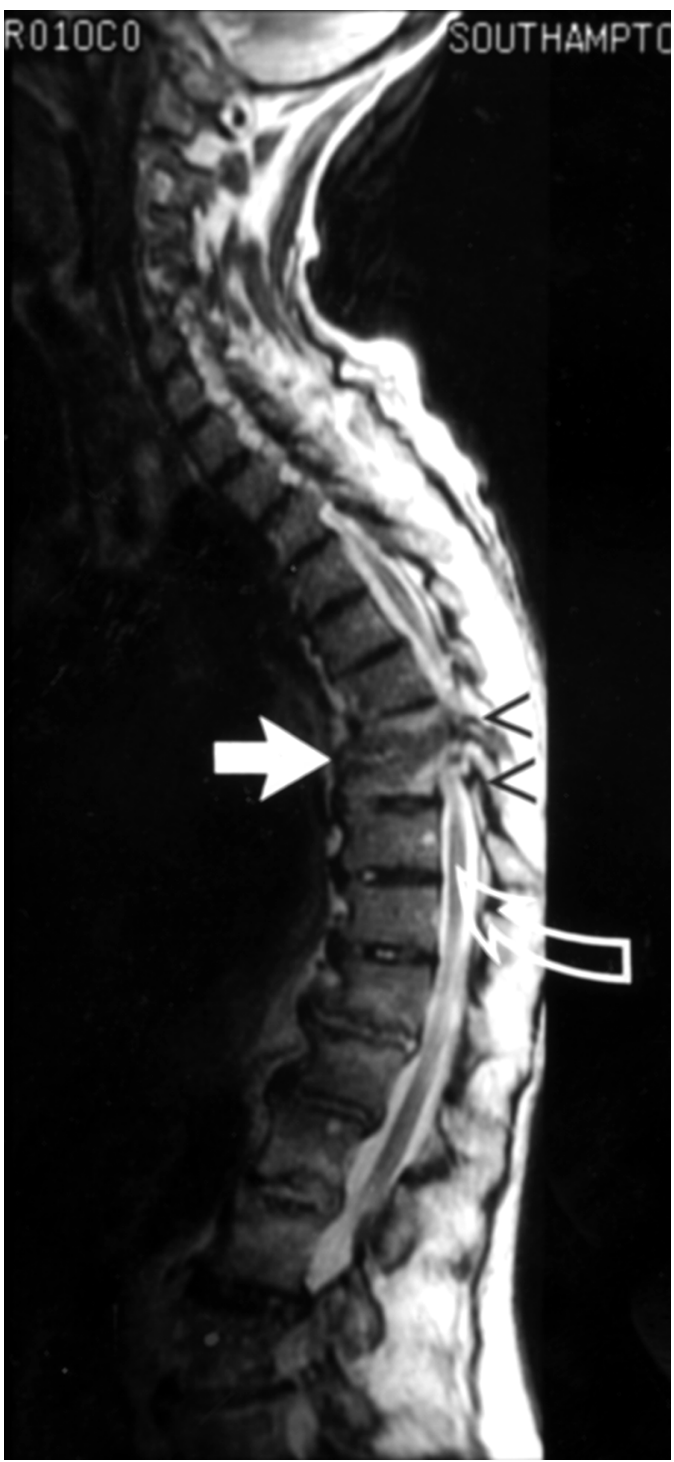

Figure 2 MRI T2 weighted sagittal section showing destruction of vertebral body and intervening disc at T6/7 (closed white arrow) with posterior extension into the spinal canal (open black arrows). Spinal cord is shown (open white arrow) as a grey filling defect surrounded by white CSF

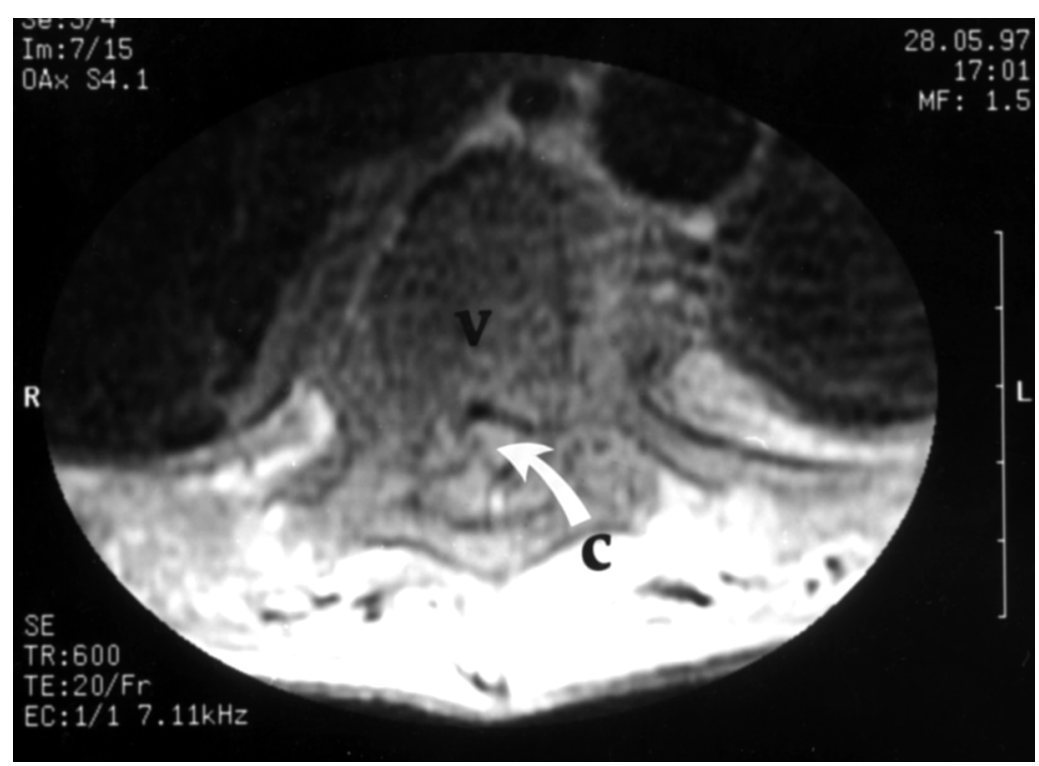

Figure 3 MRI T1 weighted transverse section showing triangularly compressed spinal cord (c) and loss of normal CSF space ( $v=$ vertebral body).
(MRI) of the spine showed abnormal signal in the T6/7 disc with collapse of the adjacent vertebral bodies and extension of abnormal material into the canal causing cord compression. This was highly suggestive of discitis with osteomyelitis and a paravertebral abscess (fig 2 and fig 3). The intensity of the back pain diminished with the onset of the paraplegia.

A neurosurgical opinion did not favour surgical decompression, as the neurological deficit did not progress. She received intensive physiotherapy and teicoplanin was continued for eight weeks. A gradual improvement became apparent with return of sensation and increase in the power in the lower limbs to grade $3 / 5$. This was reflected by the decrease in the ESR to $30 \mathrm{~mm} 1$ st hour and CRP to 27 $\mathrm{mg} / \mathrm{l}$. Repeat MRI showed marked reduction in the abnormal signal and size of the paraspinal mass, as well as resolution of the cord oedema. The patient received intensive rehabilitation in another hospital and at review after six months walks with support.

\section{Discussion}

We have reported on an elderly lady with vertebral osteoporosis who initially presented with back pain. The presence of bacterial endocarditis complicated the clinical picture and the eventual diagnosis of vertebral osteomyelitis was not apparent until she developed the paraplegia. This case illustrates several important points about back pain in vertebral osteoporosis that will be discussed: (a) differential diagnosis; (b) epidemiology of vertebral fractures and back pain; (c) management of vertebral fractures; (d) osteoporosis and cord compression; (e) causes and presentation of vertebral osteomyelitis; (f) treatment and outcome of vertebral osteomyelitis; (g) osteoporosis and osteomyelitis; (h) radiological imaging of spinal infections.

\section{Differential diagnosis}

In an elderly person presenting with back pain and one or more compression fractures the differential diagnosis includes vertebral osteoporosis, malignancy, and infection. The back pain in vertebral metastases or osteomyelitis is unrelieved by rest and may be worse at night. It increases in intensity over time because of the increased intramedullary tension, which may be relieved after cord compression as seen in our patient. Systemic features like anorexia and weight loss usually signify a sinister cause. There may be clinical clues of a septic focus (for example, endocarditis) or a primary malignancy.

An increase in acute phase reactants, leucocytosis, and positive blood cultures favour osteomyelitis, but were attributed to endocarditis in our patient. Hypercalcaemia without significant increase in alkaline phosphatase may indicate either osteolytic metastases or myeloma. Serum or urine paraprotein will also be present in the latter.

The plain radiograph shows destruction of the vertebral body in both infection and malignancy but involvement of the disc space is characteristic of the former. The radiographic 
changes take time to appear, as greater than $30 \%$ of bone must be destroyed before a lesion is evident on the radiograph. ${ }^{1}$ It also may be difficult to interpret radiographs in the presence of a spinal deformity, as in our patient. Bone scintigraphy is sensitive but not specific, showing increased uptake in metastases, infection or compression fractures. It is often negative in myeloma as the osteolytic lesions may not stimulate osteoblastic activity. Computed tomography (CT) and MRI are more sensitive and specific, showing early changes and also the extent of disease. It may sometimes be difficult to differentiate between metastatic disease and compression fracture from signal intensity changes on the $\mathrm{MRI}^{2}$ but it is the investigation of choice for vertebral osteomyelitis. $^{3}$

Bone biopsy is essential to identify the organism in vertebral osteomyelitis; it also may confirm myeloma or give clues to a primary malignancy. Osteoporosis is quantified by bone densitometry.

\section{Vertebral osteoporosis}

EPIDEMIOLOGY

Recent cross sectional and longitudinal studies have helped define the epidemiology of vertebral fracture. It has been estimated that one in four postmenopausal white women in the United States ${ }^{4}$ and one in eight in Europe have radiographic evidence of at least one vertebral deformity. ${ }^{5}$ These comparative figures are now available, following the replacement of subjective assessment of spinal radiographs by morphometric measurement of vertebral deformity. ${ }^{6}$ It is estimated that a third of all vertebral fractures come to medical attention and about $8 \%$ need hospitalisation. ${ }^{7}$ Although the impact of a single fracture may be minimal, the effects of multiple fractures are cumulative, resulting in acute and chronic back pain, limitation of physical activity, progressive kyphosis, and height loss.

In the European Vertebral Osteoporosis Study (EVOS), subjects who had three or more vertebral deformities, were twice as likely as subjects without deformity to have reported back pain in the last year. ${ }^{8}$ There was also a highly significant linear trend between the risk of reported back pain and the number of deformities. Similarly, in a study of 55-93 year old Japanese-American women in Hawaii ${ }^{9}$ severe back pain was noted in women with multiple, severe vertebral deformities. The back pain was associated only with vertebral fractures that had occurred in the previous four years and there was a sharp increase in its frequency in women over 80 years of age.

MANAGEMENT

The treatment of a new osteoporotic fracture has several elements. Immobilisation is advocated in the initial week followed by rapid functional rehabilitation. This involves lifestyle modification, physical therapy, and fitness training. ${ }^{10}$ Adequate analgesia is essential for early mobilisation. Simple analgesics are preferred to narcotics, as they have fewer side effects. Sedatives and muscle relaxants may also be useful. Physical treatments like local heat are not only useful to relieve the acute pain, but also serve to reassure the patient and encourage mobilisation. There is some evidence of a central analgesic effect of calcitonin ${ }^{11}$ administered subcutaneously (50$100 \mathrm{IU}$ on alternate days). The patient should be warned of the possible side effects of flushing and irritation at the injection site. Intravenous pamidronate is known to relieve bone pain besides increasing the bone density. ${ }^{12}$ Percutaneous vertebroplasty is a new alternative, where polymethylmethacrylate is injected into an affected vertebral body under the guidance of CT/fluoroscopy. ${ }^{10}$ It prevents vertebral body compression and also provides pain relief as shown in a small study. ${ }^{13}$ Prevention of further bone loss should be attempted and risk factors for falls in the elderly considered.

OSTEOPOROTIC COLLAPSE AND SPINAL CORD COMPRESSION

Neurological deficit after an osteoporotic vertebral collapse although rare, has been reported by a number of authors. ${ }^{14-17}$ But you should exclude disorders like myeloma, osteolytic secondaries and osteomyelitis that may also present with an osteoporotic spine and myelopathy. The anterior wedging of the vertebra, characteristic of osteoporosis, usually spares the cord. But, unstable burst fractures of the spine frequently lead to neurological deficit, as they involve the posterior vertebral body. ${ }^{15}$ They usually follow significant trauma, but sometimes may be spontaneous. The osteoporotic collapse commonly occurs between T5 and T8. This corresponds to the critical vascular zone of the spinal cord extending from $\mathrm{T} 5$ to $\mathrm{T} 9 .{ }^{14} \mathrm{~A}$ vascular factor may aggravate the mechanical compression in this region.

In a retrospective series, all nine patients gradually developed severe radicular pain and leg weakness from 1-12 weeks after the osteoporotic fracture. ${ }^{17}$ Early decompression and stabilisation produces substantial neurological improvement in the patients with severe deficit. ${ }^{17} 18$

\section{Vertebral osteomyelitis}

CAUSES AND PRESENTATION

Vertebral osteomyelitis is uncommon in healthy adults and its vague presentation may lead to a delay in diagnosis. Sapico and Montgomerie reported that more than $50 \%$ of the patients reviewed had a delay of over three months before diagnosis. ${ }^{19}$ The diagnosis of tuberculous or brucellar vertebral osteomyelitis takes longer. ${ }^{20}$

The vertebral infection is usually haematogenous except in postoperative discitis. It is common in the sixth or seventh decade with a 2:1 male predominance. ${ }^{19}$ Back pain is the presenting complaint in over $85 \%$ of the cases, but only $30-50 \%$ have fever. ${ }^{19}{ }^{21}$ Most of the patients have an increase in ESR while leucocytosis is less frequent. ${ }^{1921}$ Blood culture is positive in only $50 \%$ of the vertebral osteomyelitis cases; bone biopsy gives a higher yield even up to $86 \% .^{19^{21} 22}$ Staphylococcus aureus is the most common pathogen in 
Table 1 Bacterial aetiology of pyogenic vertebral osteomyelitis

\begin{tabular}{lll}
\hline & $\begin{array}{l}\text { Total \% of } \\
\text { culture (+) }\end{array}$ & Cases \\
Organism & & 68 \\
\hline Gram positive cocci & 57 & \\
$\quad$ Staphylococcus aureus & 4.1 & \\
Streptococcus pyogenes & 3.4 & \\
Staphylococcus, coagulase negative & 2.0 & \\
Other streptococci & 0.7 & \\
Enterococci & & \\
Gram negative bacilli & 10.5 & \\
Escherichia coli & 6.7 & \\
Proteus spp & 5.7 & \\
Pseudomonas aeruginosa & 1.8 & \\
Klebsiella pneumoniae & 1.8 & \\
Enterobacter spp & 1.8 & \\
Salmonella spp & 1.8 & \\
Serratia marcescens & & \\
Anaerobic bacteria & 2.0 & \\
Propionibacterium spp & 0.5 & \\
Bacteroides fragilis & 0.5 & \\
Peptostreptococcus spp & & \\
\hline
\end{tabular}

Data from Sapico FL, Montgomerie JZ. Pyogenic vertebral osteomyelitis: Report of nine cases and review of literature. Rev Infect Dis 1979;1:754-76.

haematogenous vertebral osteomyelitis seen in over $55 \%$ of the reported cases. ${ }^{19}{ }^{21-23}$ Table 1 shows the distribution of the bacterial aetiology of vertebral osteomyelitis. There is only one known case of a spinal epidural abscess with Streptococcus mitis in the literature. ${ }^{24}$ Our patient had vertebral osteomyelitis associated with an epidural abscess caused by Streptococcus mitis.

Tuberculosis was seen in about $30 \%$ of vertebral osteomyelitis cases, predominantly in native Indians in a Canadian study. ${ }^{23}$ It is still common in developing countries and is also increasingly recognised in the West, especially in regions with large immigrant populations. This is supported by a recent review of vertebral osteomyelitis from France, which found $M$ tuberculosis in $39 \%$ of the 80 cases. $^{25}$ The thoracic spine is a common site of extrapulmonary tuberculosis, probably because of lymphatic spread from the mediastinal nodes or the pleura. ${ }^{26}$ Lesions affecting the posterior portion of the vertebral body and paravertebral abscesses are characteristic of mycobacterial infection. In contrast with vertebral osteomyelitis, in tuberculous osteomyelitis, fever is less common, but spinal deformity and neurological deficit are more frequent. ${ }^{20}$

Brucellosis produces spinal osteomyelitis in $6-12 \%$ cases and is commonly seen in the Mediterranean region and parts of Latin America. ${ }^{20}$ Blood culture or serology usually confirms the diagnosis. A bone biopsy was needed in only $4 \%$ of the 105 cases in a recent study from Spain. ${ }^{20}$

Vertebral osteomyelitis most commonly affected the lumbar spine $(50 \%)$ followed by thoracolumbar junction (25\%), the thoracic spine $(23 \%)$, and cervical spine $(5 \%) .{ }^{23}$ An underlying cause for infection was often not found, but predisposing factors included infected intravenous cannulation sites, genitourinary tract instrumentation, diabetes, immunosuppression, and old age. ${ }^{1923} 27$ Intravenous drug users also had a higher risk of vertebral osteomyelitis, but they were usually younger and were more likely to be infected with Gram negative bacteria or fungi. ${ }^{28}$ Endocarditis pre-

\section{Key points}

- Unrelenting back pain in osteoporosis should prompt a search for another cause, especially in the presence of a septic focus.

- Vertebral osteomyelitis in the elderly may present insidiously without fever but back pain is usually prominent.

- Spinal cord compression is rare in osteoporotic vertebral collapse and is usually attributable to alternative disorder.

- MRI enables early diagnosis of vertebral osteomyelitis.

ceding vertebral osteomyelitis, as in our patient, has been described previously. ${ }^{27}$

\section{COMPLICATIONS}

Paraplegia is a well known complication, which signifies uncontrolled spread of the infection from the constraints of the vertebral body into the spinal canal. Neurological involvement has been reported in $4-40 \%$ of patients. ${ }^{23}{ }^{29}$ Other complications include psoas or pharyngeal abscess depending on the location.

TREATMENT AND OUTCOME

The duration of treatment recommended for pyogenic vertebral osteomyelitis varies widely in the medical literature, but parenteral antibiotics for at least four weeks followed by oral antibiotics for three months is considered optimal. ${ }^{19} 21272930$ The choice depends on the local sensitivity pattern, but should cover staphylococcus and Gram negative organisms if cultures are negative. The ESR is very useful in guiding the duration of treatment, but $\mathrm{C}$ reactive protein may be more sensitive as it changes rapidly. Tuberculosis and brucellosis need prolonged treatment with specific agents.

Surgery is indicated if there is progressive neurological deficit or failure to respond to medical treatment. It may also be necessary if a needle biopsy is unsuccessful or if there is spinal instability. Anterior decompression and stabilisation is preferred and significant neurological recovery from incomplete paraplegia is often noted. ${ }^{19}{ }^{27}$ There are reports of successful non-surgical treatment of patients with spinal epidural abscess associated with vertebral osteomyelitis. ${ }^{31}$

Relapse of the spinal infection is seen in $3-40 \%$ of the cases, especially if antibiotics are stopped earlier than four weeks. ${ }^{21} 22$ Spinal deformity is a common sequel. The mortality rate of vertebral osteomyelitis is less than $5 \% .{ }^{19}$

\section{Osteomyelitis with osteoporosis}

It is often less well appreciated that vertebral osteomyelitis may present as a vertebral collapse, contributing to significant delay in diagnosis and treatment, besides increasing the risk of long term morbidity. In one case series of six patients with osteoporosis and osteomyelitis, ${ }^{32}$ this presentation accounted for $13 \%$ of all hospitalised patients with vertebral 
osteomyelitis and $2.4 \%$ of inpatients with osteoporotic compression fractures in the preceding five years. It is possible that a previously fractured vertebral body represents a favourable environment for infection, as associated haemorrhage and bony debris may provide a good culture medium for bacteria. Alternatively, infection itself, aided by osteoporosis, may cause collapse of the vertebra.

\section{Radiological imaging of spinal infections}

The diagnosis of skeletal infection may entail a variety of imaging methods. Plain radiographs, show no specific changes until florid disc space destruction has occurred, which is relatively late. ${ }^{1}{ }^{99 m}$ Tc phosphate scintigraphic scanning usually shows increased uptake within 24 hours but is very non-specific; additional Gallium citrate scan improves the yield. Both CT and MRI have excellent resolution and can reveal bone destruction and soft tissue involvement even when plain radiographs are normal. MRI is the imaging technique of choice, though CT is very useful in guiding a needle biopsy. Typical MRI changes include evidence of bone marrow oedema on $\mathrm{T} 1$ and $\mathrm{T} 2$ weighted images and uptake of gadolinium producing enhancement. $^{23}$ MRI diagnosis of vertebral osteomyelitis could be made as early as two weeks from onset, enabling earlier treatment and better outcome. ${ }^{3}$

\section{Conclusion}

We have reported an elderly lady with vertebral fracture and back pain attributable to vertebral osteomyelitis associated with bacterial endocarditis. Increased awareness and a high index of suspicion would enable early diagnosis of this atypical presentation preventing significant morbidity.

1 Ardan GM. Bone destruction not demonstrable by radiography. Br J Radiol 1951:24:107-9.

2 Tan SB, Kozak JA, Mawad ME. The limitations of magnetic resonance imaging in the diagnosis of pathologic vertebral fractures. Spine 1991;8:919-23.

3 Carragee E, Kaiser J. The clinical use of magnetic resonance imaging in pyogenic vertebral osteomyelitis. Spine 1997; $780-5$

4 Ettinger B, Black DM, Nevitt MC, Rundle AC, Canley JA Cummings SR, et al. Contribution of vertebral deformities to chronic back pain and disability. J Bone Miner Res 1992; 7:449-56.

5 O'Neill TW, Felsenberg D, Varlow J, Cooper C, Kanis JA Silman AJ, et al. The prevalence of vertebral deformity in European men and women: The European Vertebral Osteoporosis Study. J Bone Miner Res 1996;11:1010-18.

6 O’Neill TW, Varlow J, Felsenberg D, Johnell O, O’Neill TW, Varlow J, et al. Variation in vertebral height ratios in population studies. J Bone Miner Res 1994; 9: 1895-907. 7 Cooper C, Melton LJ. Vertebral fractures: How large is the

8 Cooper C, O'Neill T W, Egger P, Varlow J, Felsenberg D Kanis JA, et al. Vertebral deformities: clinical impact and relation to fractures at other sites. J Bone Miner Res 1995 10:S145.

9 Huang C, Ross PD, Wasnich RD. Vertebral fractures and other predictors of back pain among older women. J Bone Miner Res 1996;11:1026-32.

10 Tamayo-Orozco J, Arzac-Palumbo P, Peon-Vidales $\mathrm{H}$ Mota-Bolfeta R, Fuentes F. Vertebral fractures associated with osteoporosis: Patient management. Am J Med with osteoporosis: 1997; 103 (2A): $44-50 \mathrm{~S}$.

11 Gennari C, Agnusdei D, Camporeale A. Use of calcitonin in the treatment of bone pain associated with osteoporosis. the treatment of bone pain associated with

12 Marshall A, Kavanagh RT, Crisp A. The effect of pamidronate on lumbar spine bone density and pain in osteoporosis secondary to systemic mastocytosis. Br J Rheumatol 1997; 36:393-6.

13 Gangi A, Kastler BA, Dietemann JL. Percutaneous vertebroplasty guided by a combination of CT and fluoroscopy. Am J Neuroradiol 1994;15:83-6.

14 Salomon C, Chopin D, Benoist M. Spinal cord compression: an exceptional complication of spinal osteoporosis. Spine 1988;13:222-4.

15 Arciero RA, Leung KYK, Pierce JH. Spontaneous unstable burst fracture of the thoracolumbar spine in osteoporosis. Spine 1989;14:114-17.

16 Tanaka S, Kubota M, Fujimoto Y, Hayashi J, Nishihawa K. Conus medullaris syndrome secondary to an L1 burst fracture in osteoporosis - a case report. Spine 1993;18:2131-4

17 Heggeness MH. Spine fracture with neurological deficit in osteoporosis. Osteoporosis Int 1993;3:215-21

18 Shikata J, Yamamuro T, Iida H, Shimizu K, Yoshikawa J. Surgical treatment for paraplegia resulting from vertebra fractures in senile osteoporosis. Spine 1990;15:485-9.

19 Sapico FL, Montgomerie JZ. Pyogenic vertebral osteomyelitis: Report of nine cases and review of literature. Rev Infect Dis 1979;1:754-76.

20 Colmenero JD, Jimenez-Mejias ME, Sanchez-Lora FJ, et al. Pyogenic, tuberculous, and brucellar vertebral Pyogenic, tuberculous, and brucellar vertebral osteomyelitis: a descriptive and comparativ
cases. Ann Rheum Dis 1997;56:709-15.

21 Torda AJ, Gottlieb T, Bradbury R. Pyogenic vertebral osteomyelitis: analysis of 20 cases and review. Clin Infect Dis 1995;20:320-8.

22 Patzakis MJ, Rao S, Wilkins J, Moore TM, Harvey PJ. Analysis of 61 cases of vertebral osteomyelitis. Clin Orthop 1991;264:178-83.

23 Joughin E, McDougall C, Parfitt C, Yong-Hing K, Kirkaldy-Willis WH. Causes and clinical management of vertebral osteomyelitis in Saskatchewan. Spine 1991;16: 261-4.

24 Martin MJ, Lee PYC. Streptococcus mitis causing epidural abscess. [Letter]. Postgrad Med J 1995;71:251.

25 Peronne J, Saba Z, Behloul D, et al. Pyogenic and tuberculous spondylodiskitis (vertebral osteomyelitis) in 80 adul patients. Clin Infect Dis 1994;19:746-50.

26 Haas DW, Des Prez RW. Mycobacterium tuberculosis. In Mandell GL, Bennett JE, Dolins R, eds. Principles and practice of infectious diseases. 4th ed. New York: Churchil Livingstone, 1995:2213-50.

27 Osenbach RK, Hitchon PW, Menezes AH. Diagnosis and management of pyogenic vertebral osteomyelitis in adults. Surg Neurol 1990;33:266-75.

28 Sapico FL, Montgomerie JZ. Pyogenic vertebral osteomyelitis in intravenous drug users: report of three cases and review of literature. Rev Infect Dis 1980;2:196-206.

29 Kern R, Houpt J. Pyogenic vertebral osteomyelitis: diagnosis and management. Can Med Assoc J 1984;130:1025-8. 30 Lew DP, Waldvogel FA. Osteomyelitis. N Engl J Med 1997; 336:999-1007.

31 Wheeler D, Keiser P, Rigamonti D, Keay S. Medical management of spinal epidural abscesses: case report and review. Clin Infect Dis 1992;15:22-7.

32 McHenry MC, Duchesneau PM, Keys TF, Rehm SJ, Boumphrey FRS. Vertebral osteomyelitis presenting as spinal compression fracture Six patients with underlyin osteoporosis. Arch Intern Med 1988;148:417-23. 